\title{
Integrated Simulation of ELM Energy Loss Determined by Pedestal MHD and SOL Transport
}

\author{
N. Hayashi, T. Takizuka, T. Ozeki, N. Aiba, N. Oyama \\ Japan Atomic Energy Agency, Naka, Ibaraki-ken, 311-0193 Japan \\ e-mail contact of main author: hayashi.nobuhiko@jaea.go.jp
}

\begin{abstract}
An integrated simulation code TOPICS-IB based on a transport code with a stability code for the peeling-ballooning modes and a scrape-off-layer (SOL) model has been developed to clarify self-consistent effects of edge localized modes (ELMs) and SOL on the plasma performance. Experimentally observed collisionality dependence of the ELM energy loss is found to be caused by both the edge bootstrap current and the SOL transport. The bootstrap current decreases with increasing the collisionality and intensifies the magnetic shear at the pedestal region. The increase of the magnetic shear reduces the width of eigenfunctions of unstable modes, which results in the reduction of the area and the edge value near the separatrix of the ELM enhanced transport. On the other hand, when an ELM crash occurs, the energy flows into the SOL and the SOL temperature rapidly increases. The increase of the SOL temperature lowers the ELM energy loss due to the flattening of the radial edge gradient. The parallel electron heat conduction determines how the SOL temperature increases. For higher collisionality, the conduction becomes lower and the SOL electron temperature increases more. By the above two mechanisms, the ELM energy loss decreases with increasing the collisionality. The bootstrap current and the SOL transport have the major effect on the collisionality dependence.
\end{abstract}

\section{Introduction}

The energy loss caused by edge localized modes (ELMs) is crucial for reducing the divertor plate lifetime and limiting the plasma confinement in tokamaks. The ELMs are considered to be induced by the high-n ballooning mode due to the large pressure gradient or by the medium-n peeling mode due to the large edge current and the pressure gradient. Characteristics of ELMs, such as the energy loss, the collapse structure and so on, have been studied by experimental measurements with the high resolution of time and space [1-4]. The analysis of ELMs from multi-machine experiments has shown that the ELM energy loss decreases with increasing the collisionality [2]. Effects of the bootstrap current and the scrape-off-layer (SOL) transport on the ELM energy loss have been discussed [2,3]. The physical mechanisms, however, are not fully understood yet. It is necessary to clarify the physical mechanism of the collisionality dependence of ELM energy loss for the projection in ITER and reactors.

The modeling of various physics elements and the integration of those models are a useful and effective method to simulate complex plasmas with various time and spatial scales. This integrated code is one of the effective methods to study the self-consistent ELM behavior [5-8], while the nonlinear simulation $[9,10]$ is a fundamental method requiring large computational resources. The integrated codes couple with ELM models, which consists of the edge transport and the stability. For example, the JETTO integrated code clarified the interplay between ballooning and peeling modes in the ELM crash [6]. These studies dealt with the ELM behavior without the dynamic response of SOL plasmas. The SOL plasma transport is, however, important for the ELM behavior because the SOL plasma affects boundary conditions of the core transport. Indeed, the edge boundary conditions at the separatrix were found to affect the ELM behavior in the integrated simulation [6]. An appropriate SOL model is required for the integrated modeling.

While 2-dimensional (2D) fluid simulations consume much calculation-time, a two-point 
model based on integral fluid equations easily reproduces many static features of SOL plasmas in experiments [11]. Such point models are suitable not only for studying physics in a wide range of parameter space but also for coupling with core transport codes [12]. We have extended the point model and developed a dynamic five-point model to study the response of the SOL plasmas to an ELM crash [13]. This five-point model can reproduce static and dynamic features obtained by particle and fluid codes.

In this paper, we develop an integrated simulation code TOPICS-IB with a stability code for the peeling-ballooning modes and a SOL model. TOPICS-IB is based on the 1.5D transport code TOPICS [14] extended to the integrated simulation for burning plasmas. The collisionality dependence of the ELM energy loss is investigated by artificially enhancing the collisionality in the bootstrap current and SOL models. In the next section, we explain TOPICS-IB. The integrated simulation result of whole plasmas during the pedestal growth and an ELM crash is shown in Section 3. The physical mechanism of the reduction of ELM energy loss through the bootstrap current and the SOL transport is clarified in Sections 4 and 5. The dependence of the ELM energy loss on the collisionality and model parameters is examined in Section 6. Conclusions are presented in the last section.

\section{Integrated code TOPICS-IB for the ELM dynamics}

The energy loss caused by an ELM crash is investigated by TOPICS-IB, in which TOPICS is coupled with the ELM model [5] and the SOL model [13].

\subsection{5D transport code TOPICS}

TOPICS self-consistently solves the 1D transport and current diffusion equations and the Grad-Shafranov equation of the MHD equilibrium on the 2D plane. The transport equations are the continuity equation for the deuterium ion density, $n_{i}$, the power balance equations for the electron temperature, $T_{e}$, and the ion temperature, $T_{i}$, on the coordinate of the normalized minor radius, $\rho$, defined by the square root of the toroidal flux. Details of TOPICS are shown in Ref.[14,15]. In this paper, thermal diffusivities are assumed as $\chi_{\mathrm{e}, \mathrm{i}}=\chi_{\mathrm{neo}, \mathrm{i}}+\chi_{\mathrm{ano}, \mathrm{e}, \mathrm{i}}$, where $\chi_{\text {neo,i }}$ denotes the neoclassical ion diffusivity calculated by the matrix inversion method [14], which also calculates the bootstrap current. The anomalous diffusivities $\chi_{\text {ano,e,i }}$ are simply given as an empirical formula, $\chi_{\text {ano }, \mathrm{e}}=0.18\left(1+2 \rho^{3}\right)\left(1+\left(\mathrm{P}_{\mathrm{NB}}[\mathrm{MW}]\right)^{0.5}\right)\left[\mathrm{m}^{2} / \mathrm{s}\right]$ and $\chi_{\text {ano,i }}=2$ $\chi_{\text {ano,e }}$. In order to produce the H-mode pedestal structure, the transport near the edge is reduced to the neoclassical level $\left(\chi_{\mathrm{ano}, \mathrm{e}, \mathrm{i}}=0\right)$ as shown in Fig.1(c) where the pedestal width, $\Delta_{\text {ped }}$, is fixed constant for simplicity.

\subsection{ELM model}

The ELM model [5] has been developed by coupling TOPICS with the linear MHD stability code MARG2D [17,18]. The MARG2D code solves the eigenvalue problem associated with the 2D Newcomb equation and is applicable to the wide range of mode numbers from low to high. In the present simulation, stabilities of $n=1-30$ modes are examined by MARG2D in each time step along the pedestal growth. When one or some modes become unstable, an ELM occurs. The diffusivity is assumed to be enhanced according to radial profiles of eigenfunctions of unstable modes as follows: $\chi_{\mathrm{e}, \mathrm{i}}=\chi_{\text {neo,i }}+\chi_{\text {ano,e, } \mathrm{i}}+\chi_{\mathrm{ELM}}$. The ELM enhanced diffusivity $\chi_{\mathrm{ELM}}$ is $\chi_{\mathrm{ELM}}=\chi_{\mathrm{ELM}}{ }^{\mathrm{max}} \times\left(\Sigma \xi_{\mathrm{r}}^{2}\right) / \mathrm{N}$, where $\chi_{\mathrm{ELM}}{ }^{\max }$ is the maximum value, $\xi_{\mathrm{r}}$ denote the radial component of the eigenfunction normalized by its maximum value, and $\mathrm{N}$ is the total number of the unstable modes. The ELM enhanced transport is maintained for a time 
interval $\tau_{\mathrm{ELM}}$ given as a parameter. Figure 1 shows example profiles of (a) the total pressure, $\mathrm{P}$, the safety factor, q, at the ELM onset, (b) $\xi_{\mathrm{r}}$ of the most unstable mode $n=17$, and (c) $\chi_{\mathrm{e}}$ before and during ELM. In this case, medium- $n(n=11-22)$ modes are unstable. The infinite- $n$ ballooning mode is stable in the whole region. The unstable modes is localized near the edge.

\subsection{SOL model}

We couple the five-point model [13] to TOPICS and investigate the self-consistent transport covering core-pedestal-SOL plasmas. The five-point model is based on the integral of time dependent fluid equations, i.e., particle, momentum, electron and ion temperatures, generalized Ohm's law and current equations. The momentum loss is not taken into account at present for simplicity. The model geometry is an open magnetic flux-tube nearest to the separatrix. The flux tube is divided into four regions: the SOL region divided into two regions at the stagnation point where the parallel particle flux is zero and two divertor regions on either side of the SOL region. The integral fluid equations in
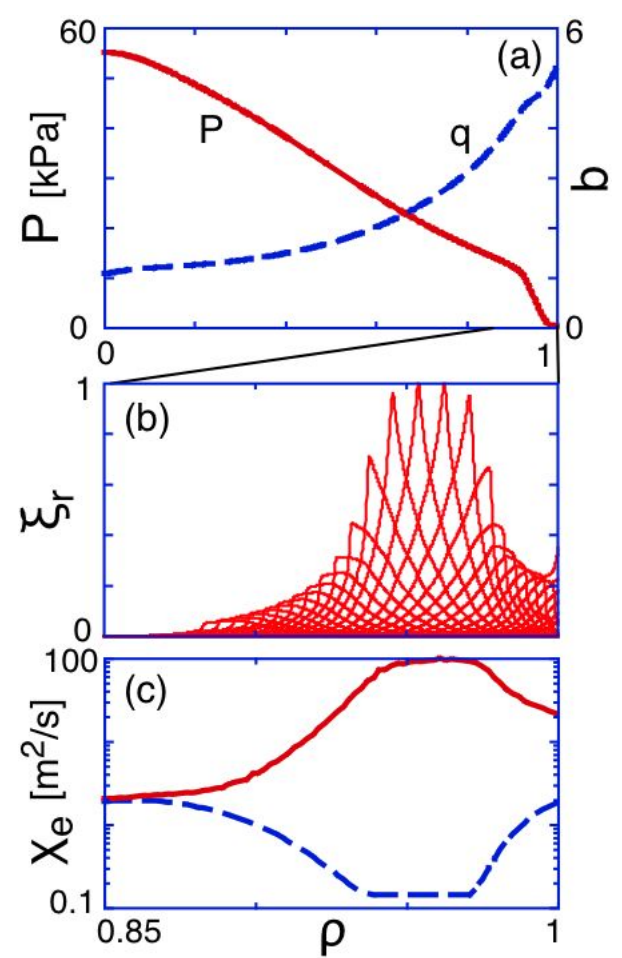

Fig.1 (a) Profiles of $P, q$ at ELM onset, (b) $\xi_{r}$ of $n=17$ and (c) $\chi_{e}$ before (broken) and during ELM (solid). each region are reduced to a set of nonlinear equations with physical variables at five positions (stagnation point, upstream throats of divertor regions and sheath entrances). Exponential radial profiles are assumed and those characteristic scale length are solved in the model. Kinetic-effect models based on the results from the particle code are introduced. Details of the five-point model are shown in Ref.[13]. The five-point model can deal with the in-out/up-down asymmtery of divertor plasmas, but the symmetry is assumed in this paper for simplicity.

The five-point model is coupled with TOPICS in the following procedure. First, radial heat and particle fluxes across the separatrix, $\mathrm{Q}_{\mathrm{r}}$ and $\Gamma_{\mathrm{r}}$, obtained in TOPICS are used as inputs for the five-point model. Next, values of the SOL plasma density at the separatrix, $\mathrm{n}_{\mathrm{iSOL}}$, and temperatures, $\mathrm{T}_{\mathrm{eSOL}}$ and $\mathrm{T}_{\mathrm{iSOL}}$, are calculated by the five-point model, which are used as boundary conditions for TOPICS in the next time step. Then, radial fluxes are recalculated and the simulation goes on. Heat and particle diffusivities in the five-point model are set equal to those values at the separatrix in TOPICS. The localization of ELMs at the low-field side midplane and the filamentary structure [9] are not taken into account in the present model, as the same in other integrated codes [5-8].

The collisionality dependence in the five-point model appears through the parallel heat conduction and the equipartition energy flow between electrons and ions. In order to clarify the effect of the SOL transport on the collisionality dependence of the ELM energy loss, the collisionality in the five-point model is artificially enhanced in Section 5.

\section{Integrated simulation result}

The ELM activity is simulated for JT-60U like parameters: $\mathrm{R}=3.4 \mathrm{~m}, \mathrm{a}=0.9 \mathrm{~m}, \kappa=1.5, \delta=0.21$, $\mathrm{I}_{\mathrm{p}}=1.5 \mathrm{MA}, \mathrm{B}_{\mathrm{t}}=3.5 \mathrm{~T}$ and $\beta_{\mathrm{N}} \sim 0.8-1$. The ELM parameters are chosen as $\tau_{\mathrm{ELM}}=200 \mu \mathrm{s}$, 


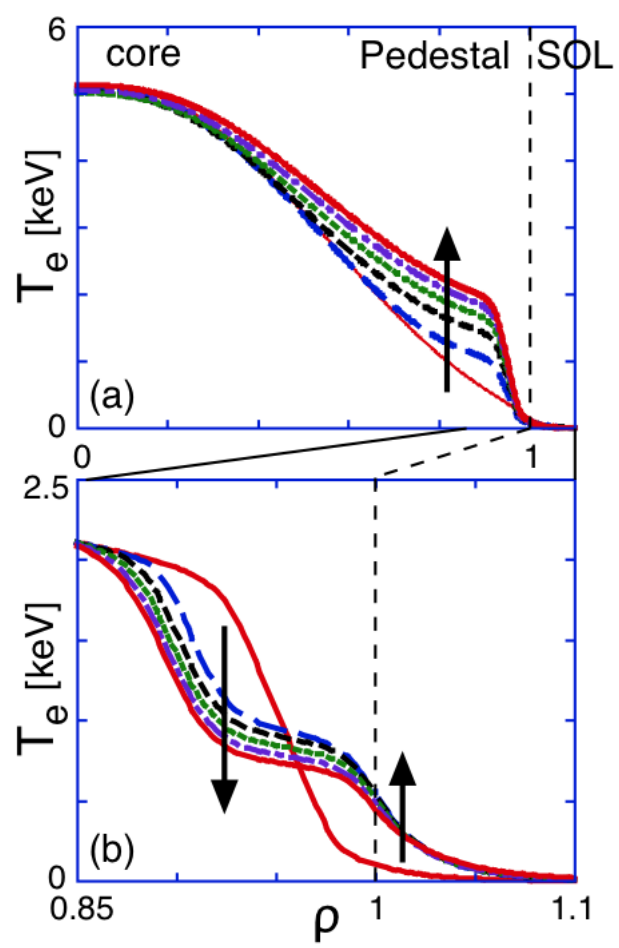

Fig.2 Time evolution of $T_{e}$ profiles (a) before and $(b)$ during an ELM crash. In (a), lines denote $30 \mathrm{~ms}$ interval from $t=0.09 \mathrm{~s}$ to $0.24 \mathrm{~s}$ in Fig.3. In (b), $40 \mu$ s interval from $t=0.24 s$ to $0.2402 s$. $\chi_{\mathrm{ELM}}{ }^{\mathrm{max}}=100 \mathrm{~m}^{2} / \mathrm{s}$ and $\Delta_{\mathrm{ped}}=0.05$. The dependence of simulation results on the ELM parameters is examined in Section 6. The collisionality dependence of ELM energy loss was found to be mainly caused by the collapse of the temperature profile in an ELM crash [2-4]. The density collapse strongly connects with the neutral recycling. In this paper, however, the density profile is fixed as $n_{i}=2.2 \times 10^{19}\left(0.675\left(1-\rho^{2}\right)^{0.5}+\right.$ $0.325)\left[\mathrm{m}^{-3}\right]\left(\mathrm{n}_{\mathrm{iSOL}}=7.2 \times 10^{18}\left[\mathrm{~m}^{-3}\right]\right)$, for simplicity. The integration of neutral models in both TOPICS and the SOL model remains as future work. The divertor plasma is assumed to be under the high recycling condition and the divertor plasma density, $\mathrm{n}_{\mathrm{idiv}}$, is chosen as $\mathrm{n}_{\mathrm{idiv}}=1 \times 10^{20}\left[\mathrm{~m}^{-3}\right]$. The effect of the recycling condition as well as the density collapse on the ELM behavior will be studied in future.

Figure 2 shows the time evolution of profiles of the electron temperature (a) before and (b) during an ELM crash. The ion temperature at the pedestal top, $\mathrm{T}_{\text {iped }} \sim 3$ $\mathrm{keV}$, is higher than the electron one $\mathrm{T}_{\text {eped }} \sim 1.8 \mathrm{keV}$ on the ELM onset. Figure 3 shows the time evolution of (a) the electron part of the stored energy, $\mathrm{W}_{\mathrm{es}}$, the ion part, $\mathrm{W}_{\text {is }}$, the total stored energy, $\mathrm{W}_{\mathrm{s}}$, (b) $\mathrm{Q}_{\mathrm{r}}$, (c) $\mathrm{T}_{\mathrm{SOL}}$ and the divertor plasma temperature, $\mathrm{T}_{\mathrm{div}}$. The simulation starts at nearly steady state and the reduction of diffusivities
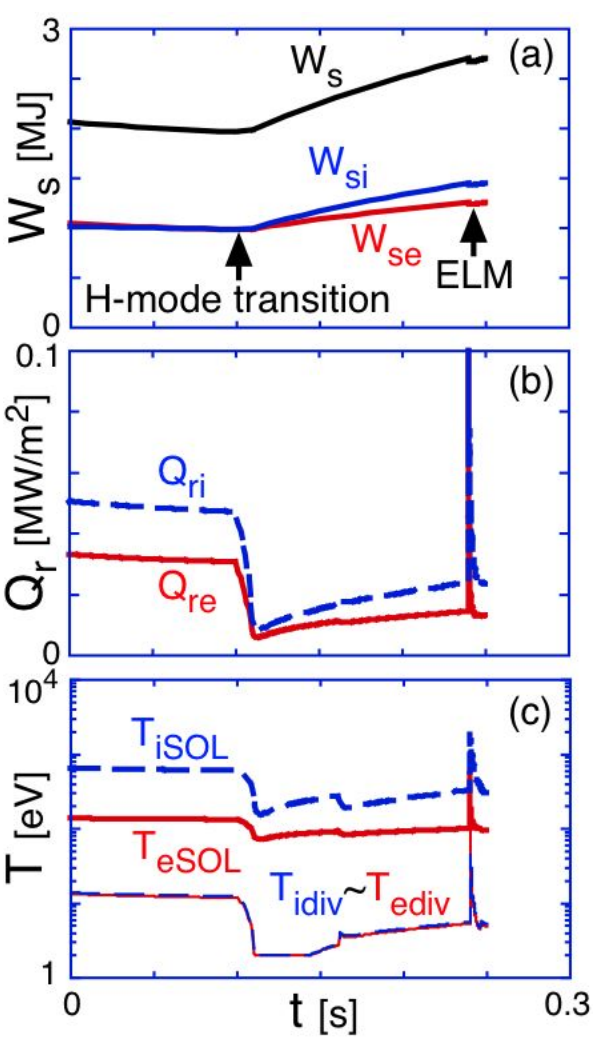

Fig. 3 Time evolution of $(a) W_{s e}, W_{s i}, W_{s},(b)$ $Q_{r e}, Q_{r i}, \quad(c) T_{e S O L}, T_{i S O L}, T_{e d i v}$ and $T_{\text {idiv }}$. rofiles are shown in Fig.2.
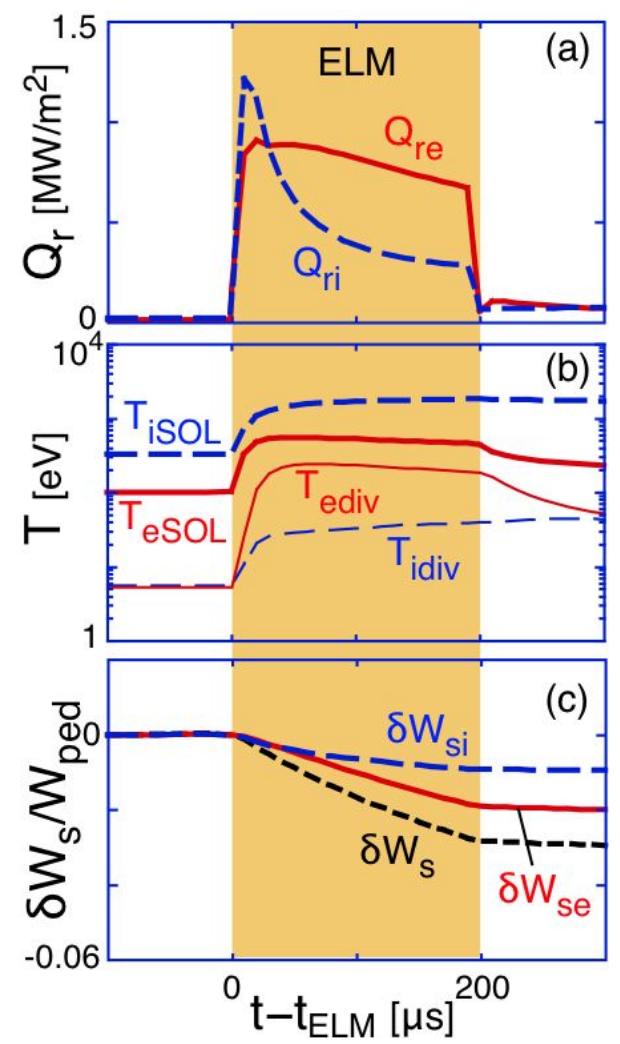

Fig.4 Time evolution of (a) $Q_{r e}, Q_{r i}$, (b) $T_{e S O L}, T_{i S O L}, T_{e d i v}, T_{i d i v},(c) \delta W_{s e}$, $\delta W_{s i}$ and $\delta W_{s}$ during an ELM crash on $t-t_{E L M}=0-200 \mu$ s (shaded region) for a simulation of Fig.3. 
for the pedestal begins at $\mathrm{t}=0.1 \mathrm{~s}$. Along the pedestal growth, the temperatures increase in the core-pedestal regions in Fig. 2(a) and the stored energies increase in Fig.3(a). The reduction of diffusivities transiently decreases the radial fluxes and the SOL-divertor plasma temperatures in Fig.3(b) and (c). The SOL ion temperature $\mathrm{T}_{\mathrm{isOL}}$ is higher than $\mathrm{T}_{\mathrm{esOL}}$, while $\mathrm{T}_{\text {idiv }} \sim \mathrm{T}_{\text {ediv }}$ due to the strong equipartition in the high-density divertor plasma.

In the progress of the pedestal growth, the finite-n modes ( $\mathrm{n}>10)$ become unstable and an ELM occurs at $\mathrm{t}$ $=\mathrm{t}_{\mathrm{ELM}}=0.24 \mathrm{~s}$. The profile of the ELM enhanced diffusivity is almost the same as that in Fig.1(c). The collapse of the temperature profile occurs in the pedestal region in Fig.2(b). The drop of $T_{e}$ at the pedestal top is about $50 \%$ of $\mathrm{T}_{\text {eped }}$ and is comparable with those measured in experiments $[3,4]$. The pedestal energy flows into the SOL plasma and the SOL-divertor temperatures rapidly increase as shown in Fig.4(a) and (b). Figure 4(c) shows the time evolution of the ELM energy loss, $\delta \mathrm{W}_{\mathrm{s}}$, normalized by the pedestal energy, $\mathrm{W}_{\text {ped }} \sim 1 \mathrm{MJ}$. The energy is lost during the ELM duration of $\tau_{\mathrm{ELM}}=200 \mu \mathrm{s}$. The resultant ELM energy loss is less than $10 \%$ of the pedestal energy and is comparable to that obtained by the diamagnetic measurement in JT-60U [1]. The electron energy loss is larger than the ion one due to larger parallel heat conduction of electrons. TOPICS-IB successfully simulates the transient behavior of the whole plasmas.

\section{Reduction of the ELM energy loss through the bootstrap current}

The bootstrap current is influenced by the collisionality and affects the pedestal MHD through the modification of the magnetic shear. We first study the collisionality effect through the bootstrap current. In this case, the SOL temperature is fixed to $100 \mathrm{eV}$ and the SOL model is not used in order to clear only the bootstrap current effect. Profiles at the ELM onset and the ELM enhanced diffusivity are almost the same as those in the case with the SOL model. Here, we define the artificially enhanced collisionality, $\mathrm{v}^{*}{ }_{\text {ped }}=\mathrm{C} \mathrm{v}^{*}$ ped 0 , where $\mathrm{C}$ is the enhancing factor in the model and $v^{*}$ ped $0(=0.08)$ is the normalized electron collisionality of the pedestal plasma. The collisionality of the bootstrap current model is artificially enhanced by $C_{B S}=100\left(v^{*}{ }_{\text {ped }}=9\right)$, where $C_{B S}$ is $\mathrm{C}$ for the bootstrap current model. Profiles of the bootstrap current, $\mathrm{j}_{\mathrm{BS}}$, and the magnetic shear, $\mathrm{s}$, just before an ELM crash are shown in Fig.5(a) and (b). The bootstrap current decreases with increasing the collisionality in Fig.5(a) and intensifies the magnetic

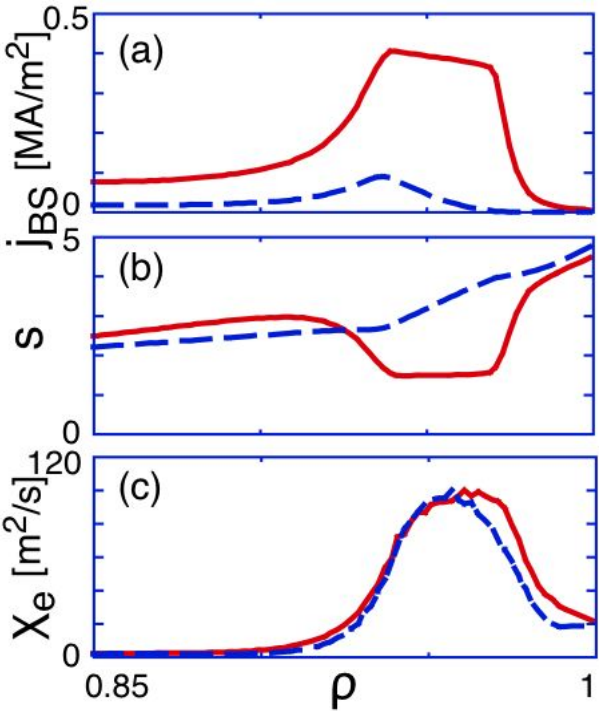

Fig.5 Profiles of (a) $j_{B S}$, (b) $s$ just before an ELM crash, (c) $\chi_{e}$ during an ELM crash for bootstrap current with $C_{B S}=1$ (solid) and 100 (broken).
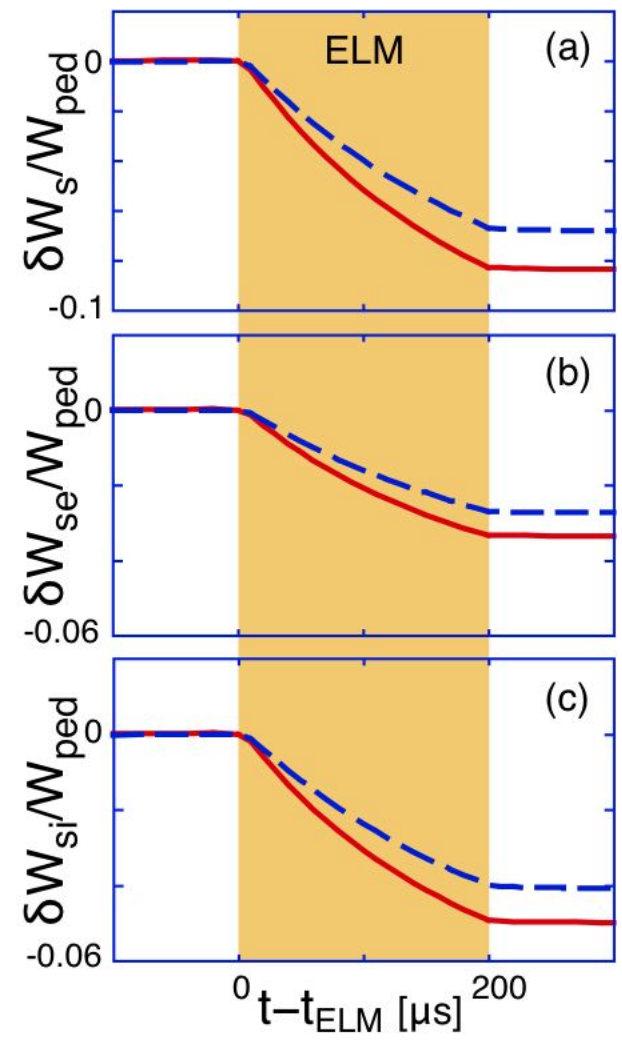

Fig.6 Time evolution of (a) $\delta W_{s},(b)$ $\delta W_{s e}$, and (c) $\delta W_{s i}$ during an ELM crash without SOL model where lines denote the same cases in Fig.5. 
shear at the pedestal region in Fig.5(b). The increase of the magnetic shear reduces the width of eigenfunctions of unstable modes. The area and the edge value near $\rho=1$ of the ELM enhanced transport are reduced through the eigenfunctions in Fig.5(c). As a result, the ELM energy loss decreases with increasing the collisionality through the bootstrap current in Fig.6(a). Both the electron and ion energy losses are reduced in almost the same ratio in Fig.6(b) and (c). Due to both the pre-ELM condition of $\mathrm{T}_{\text {iped }}>\mathrm{T}_{\text {eped }}$ and the fixed SOL temperatures, the ion energy loss is larger than the electron one, which is different from the case with the SOL model (compare Fig.6(b)(c) with Fig.4(c)). The total ELM energy loss is about three times larger than that in the case with the SOL model (compare Fig.6(a) with Fig.4(c)). These differences indicate the importance of the SOL plasma in the behavior of the ELM energy loss.

\section{Reduction of the ELM energy loss through the SOL transport}

The effect of the SOL plasma transport on the ELM energy loss is investigated. When an ELM crash occurs, the SOL plasma temperature at $\rho=1$ rapidly increases due to the energy flow from the pedestal region in Fig.4(b). The increase of the SOL temperature mitigates the radial edge gradient in Fig.2(b) and lowers the ELM energy loss. This results in the difference between the energy loss with and without the SOL transport, as mentioned in the previous section. The parallel heat conduction, which is inversely proportional to the collisionality, determines how the SOL temperatures increase. In order to study the collisionality effect through the SOL transport, the collisionality in the SOL model is artificially enhanced by $\mathrm{C}_{\mathrm{SOL}}=100\left(\mathrm{v}^{*}\right.$ ped $\left.=9\right)$. When the collisionality in the SOL model is enhanced, the SOL temperatures vary before the ELM onset in Fig.7. The profile of the ELM enhanced diffusivity as well as the eigenfunctions does not change much. For higher collisionality, the conduction becomes lower and the SOL electron temperature increases more in Fig.7(a). On the other hand, the SOL ion temperature decreases with increasing the collisionality because the equipartition energy flows from ions to electrons is proportional to the collisionality. As a result, the electron energy loss decreases with increasing the collisionality in Fig.8(b). The ion energy loss increases a little compared with the reduction of the electron one in Fig.8(c). Therefore, the total energy loss is reduced according to the electron energy loss in Fig.8(a). The ELM energy loss is reduced through the SOL transport as well as the bootstrap current mentioned in the
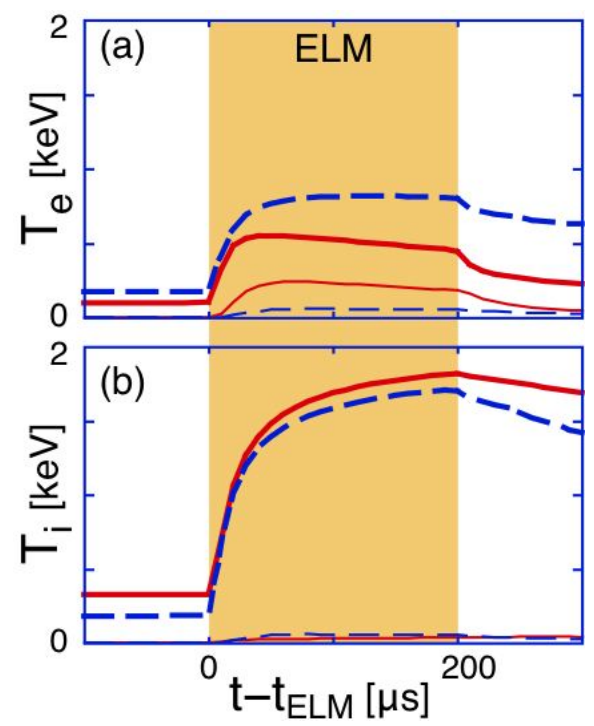

Fig.7 Time evolution of (a) $T_{e S O L}$, $T_{\text {ediv }}$ (b) $T_{\text {iSOL }}$ and $T_{\text {idiv }}$ during an ELM crash for SOL plasma with $C_{S O L}=1$ (solid) and 100 (broken). Thick and thin lines denote SOL and divertor plasmas.

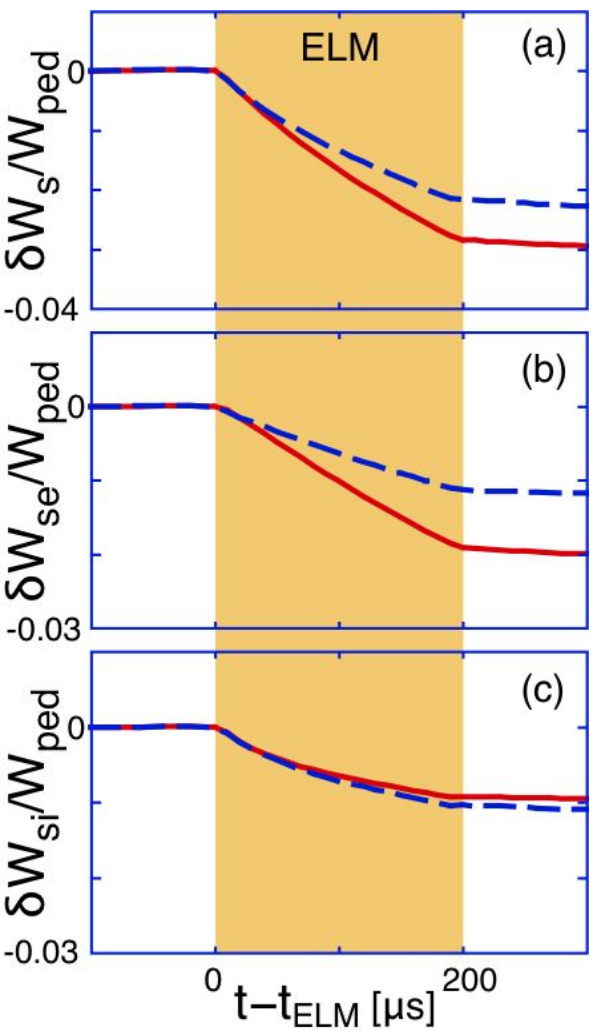

Fig.8 Time evolution of (a) $\delta W_{s},(b)$ $\delta W_{\text {se }}$, and (c) $\delta W_{\text {si }}$ during an ELM crash with SOL plasma response where lines denote the same cases in Fig. 7. 
previous section.

\section{Dependence of ELM energy loss on the collisionality and the model parameters}

We investigate the collisionality dependence of the energy loss caused by both effects of the bootstrap current and the SOL transport $\left(\mathrm{C}=\mathrm{C}_{\mathrm{BS}}=\mathrm{C}_{\mathrm{SOL}}\right)$. Figure 9 shows the magnitude of the ELM energy loss, $\Delta \mathrm{W}_{\mathrm{ELM}} / \mathrm{W}_{\text {ped }}$, as a function of $v^{*}{ }_{\text {ped }}$ for $\mathrm{C}=1,10,100$. When the collisionality is enhanced by $\mathrm{C}=100\left(\mathrm{v}_{\text {ped }}^{*}=9\right)$, the electron energy loss is reduced by $1 / 3$. The ion energy loss decreases a little with increasing the collisionality. The bootstrap current reduces the ion energy loss as shown in Fig.6(c), but the SOL transport enhances it as shown in Fig.8(c) and mitigates the reduction. Both the sensitivity of the electron energy loss to the collisionality and the insensitivity of the ion one were also observed in DIII-D experiments [4]. As a result, the total energy loss is reduced about half in Fig.9.

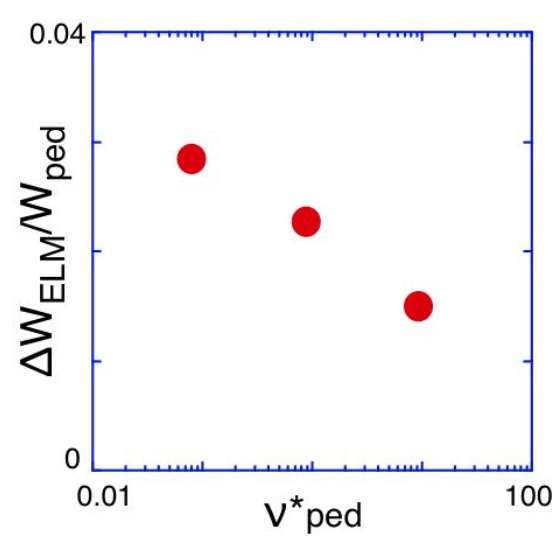

Fig. 9 Dependence of $\Delta W_{E L M} / W_{\text {ped }}$ on $v^{*}$ ped.

We next examine the dependence of the ELM energy loss on the following model parameters. In the simulations up to here, the parameters, $\tau_{\mathrm{ELM}}=200 \mu \mathrm{s}, \chi_{\mathrm{ELM}}{ }^{\max }=100 \mathrm{~m}^{2} / \mathrm{s}$ and $\Delta_{\text {ped }}=0.05$, are used as a standard case. Table 1 shows the dependence of $\Delta \mathrm{W}_{\mathrm{ELM}} / \mathrm{W}_{\text {ped }}$ on the parameters where $\mathrm{C}=1\left(v^{*}{ }_{\mathrm{ped}}=0.08\right)$ and one of $\tau_{\mathrm{ELM}}, \chi_{\mathrm{ELM}}{ }^{\mathrm{max}}$ and $\Delta_{\text {ped }}$ is multiplied twice in each case. In the case of $\Delta_{\text {ped }} \times 2$, the scale length is multiplied twice and the ELM enhanced transport is also multiplied by 4. As shown in Table 1, the ELM energy loss is enhanced by increasing the ELM duration, the ELM enhanced diffusivity and the pedestal width. The value of $\Delta \mathrm{W}_{\text {ELM }} / \mathrm{W}_{\text {ped }} \sim 0.05$ was experimentally obtained in JT-60U plasmas with the low collisionality $\left(v^{*}{ }_{\text {ped }} \sim 0.1\right)[1]$.

Table 1: Dependence of $\left|\delta W_{s}\right| / W_{\text {ped }}$ on model parameters.

\begin{tabular}{|c|c|c|c|c|}
\hline Case & Standard & $\tau_{\mathrm{ELM}} \times 2$ & $\chi_{\mathrm{ELM}}{ }^{\max } \times 2$ & $\begin{array}{c}\Delta_{\text {ped }} \times 2 \& \\
\chi_{\text {ELM }}{ }^{\max } \times 4\end{array}$ \\
\hline$\Delta \mathrm{W}_{\mathrm{ELM}} / \mathrm{W}_{\text {ped }}$ & 0.028 & 0.047 & 0.040 & 0.049 \\
\hline
\end{tabular}

The density collapse, which is not considered in this paper, enhances the ELM energy loss by about $40 \%$ under the assumption of the similar collapse to the temperature one. As shown in Fig.5(a), the bootstrap current is small near the separatrix. When the bootstrap current increases near the separatrix, the unstable mode number is varied from medium-n $(n>10)$ to low-n $(n<10)$ but the localization of eigenfunctions near the pedestal region does not change. The bootstrap current near the separatrix does not change the ELM energy loss very much.

Although the magnitude of the ELM energy loss is changed by the values of parameters in Table 1, the collisionality dependence of the ELM energy loss through the bootstrap current and the SOL transport in Fig.9 is unchanged. For example, the value of $\left|\delta \mathrm{W}_{\mathrm{s}}\right| / \mathrm{W}_{\text {ped }}$ is reduced from 0.047 to 0.025 by $\mathrm{C}=100\left(v^{*}{ }_{\text {ped }}=9\right)$ in the case of $\tau_{\mathrm{ELM}} \times 2$ on Table 1 . The reduction obtained in the simulation is not enough large compared with that obtained experimentally where the ELM energy loss is reduced by about $1 / 3$ in the same range of $v^{*}$ ped in Fig.9. However, the bootstrap current and the SOL transport have the major effect on the collisionality dependence. 


\section{Conclusion}

We have developed an integrated simulation code TOPICS-IB based on a 1.5D transport code with a stability code for the peeling-ballooning modes and a SOL model to clarify self-consistent effects of ELMs and SOL on the plasma performance. Experimentally observed collisionality dependence of the ELM energy loss is found to be caused by both the edge bootstrap current and the SOL transport. The bootstrap current decreases with increasing the collisionality and intensifies the magnetic shear at the pedestal region. The increase of the magnetic shear reduces the width of eigenfunctions of unstable modes, which results in the reduction of the area and the edge value near the separatrix of the ELM enhanced transport. On the other hand, when an ELM crash occurs, the energy flows into the SOL and the SOL temperature rapidly increases. The increase of the SOL temperature lowers the ELM energy loss due to the flattening of the radial edge gradient. The parallel electron heat conduction determines how the SOL temperature increases. For higher collisionality, the conduction becomes lower and the SOL electron temperature increases more. By the above two mechanisms, the ELM energy loss decreases with increasing the collisionality. The bootstrap current and the SOL transport have the major effect on the collisionality dependence.

The simulation and the model validation on other sets of parameters, including those of other tokamaks, remains as future work. The different plasma shape may affect the ELM energy loss through the change of the mode structure. The effect of the plasma shape on the ELM energy loss should be examined. The model improvement, such as the density dynamics, the pedestal width and so on, will be done.

Acknowledgements: We would like to thank Dr. R. Hiwatari for fruitful discussion and comments and Mr. I. Kamata for developing and running the integrated code. We are grateful to JT-60 team members for collaboration. This work was partly supported by JSPS, Grant-in-Aid for Scientific Research.

\section{References}

[1] OYAMA, N., et al., Nucl. Fusion 44 (2004) 582.

[2] LOARTE, A., et al., Plasma Phys. Control. Fusion 45 (2003) 1549.

[3] LEONARD, A.W., et al., J. Nucl. Mater. 313-316 (2003) 768.

[4] WADE, M.R., et al., Phys. Plasmas 12 (2005) 056120.

[4] OZEKI, T., et al., Fusion Sci. Tech. 50 (2006) 68.

[5] ONJUN, T., et al., Phys. Plasmas 12 (2005) 012506.

[6] LONNROTH, J.S., et al., Contrib. Plasma Phys. 46 (2006) 726.

[7] PANKIN, A. Y., et al., Nucl. Fusion 46 (2006) 403.

[8] SNYDER, P.B., et al., Phys. Plasmas 12 (2005) 056115.

[9] BEYER, P., et al., Phys. Rev. Lett. 94 (2005) 105001.

[10] STANGEBY, P.C., The plasma boundary of magnetic fusion devices, Institute of physics publishing, Bristol and Philadelphia, 2000.

[11] HIWATARI, R., et al., J. Nucl. Mater. 337-339 (2005) 386.

[12] HAYASHI, N., et al., "Modeling of dynamic response of SOL-divertor plasmas to an ELM crash", submitted to J. Nucl. Mater.

[13] SHIRAI, H., et al., Plasma Phys. Control. Fusion 42 (2000) 1193.

[14] HAYASHI, N., et al., J. Plasma Fusion Res., 80 (2004) 605.

[15] KIKUCHI, M., et al., Nucl. Fusion, 30 (1990) 343.

[16] TOKUDA, S., et al., Phys. Plasmas 6 (1999) 3012.

[17] AIBA, N., et al., this conference TH/P8-1. 